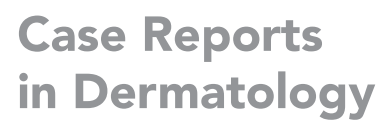

\title{
GFAP-Negative Subcutaneous Sacrococcygeal Extraspinal Ependymoma
}

\author{
Kazuya Goto ${ }^{a, b}$ Hiroko Fujii ${ }^{a, b}$ Gen Honjo ${ }^{c, d}$ Satoshi Kore-eda ${ }^{a}$ e \\ ${ }^{a}$ Department of Dermatology, Tenri Hospital, Tenri, Japan; ${ }^{b}$ Department of Dermatology, \\ Graduate School of Medicine, Kyoto University, Kyoto, Japan; 'Department of Diagnostic \\ Pathology, Tenri Hospital, Tenri, Japan; dDepartment of Diagnostic Pathology, Kitano

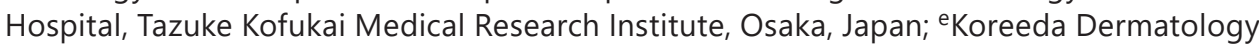 \\ Clinic, Kyoto, Japan
}

\section{Keywords}

Ependymoma - Glial fibrillary acidic protein - Subcutaneous tumor - Sacrococcygeal tumor

\begin{abstract}
Ependymomas are slowly growing glial tumors derived from the ependymal cells and usually occur in the central nervous system (CNS). Ependymomas rarely occur outside of the CNS and they are called extraspinal ependymomas. In spite of their metastatic potential, extraspinal ependymomas can be misdiagnosed for other benign mass like pilonidal cysts. The diagnosis is confirmed by histopathology and most of the cases are known to show glial fibrillary acidic protein (GFAP), S-100 protein, and keratin (AE1AE3) immunoreactivity. Herein, we present a case of GFAP-negative ependymoma, which presented as asymptomatic subcutaneous tumor of the left buttock and was clinically misdiagnosed as epidermal cyst. Our case indicates that ependymomas cannot be ruled out by lack of GFAP immunoreactivity and an asymptomatic subcutaneous mass could be a malignant tumor like ependymomas, which requires careful examinations.
\end{abstract}

\section{Introduction}

Ependymomas are slowly growing glial tumors derived from the ependymal cells and usually occur in the central nervous system (CNS) of children and young adults. They account for only $6 \%$ of intracranial gliomas, but they are the most common tumors in the spinal cord, accounting for over $60 \%$ [1]. Ependymomas rarely occur outside of the CNS and they are 


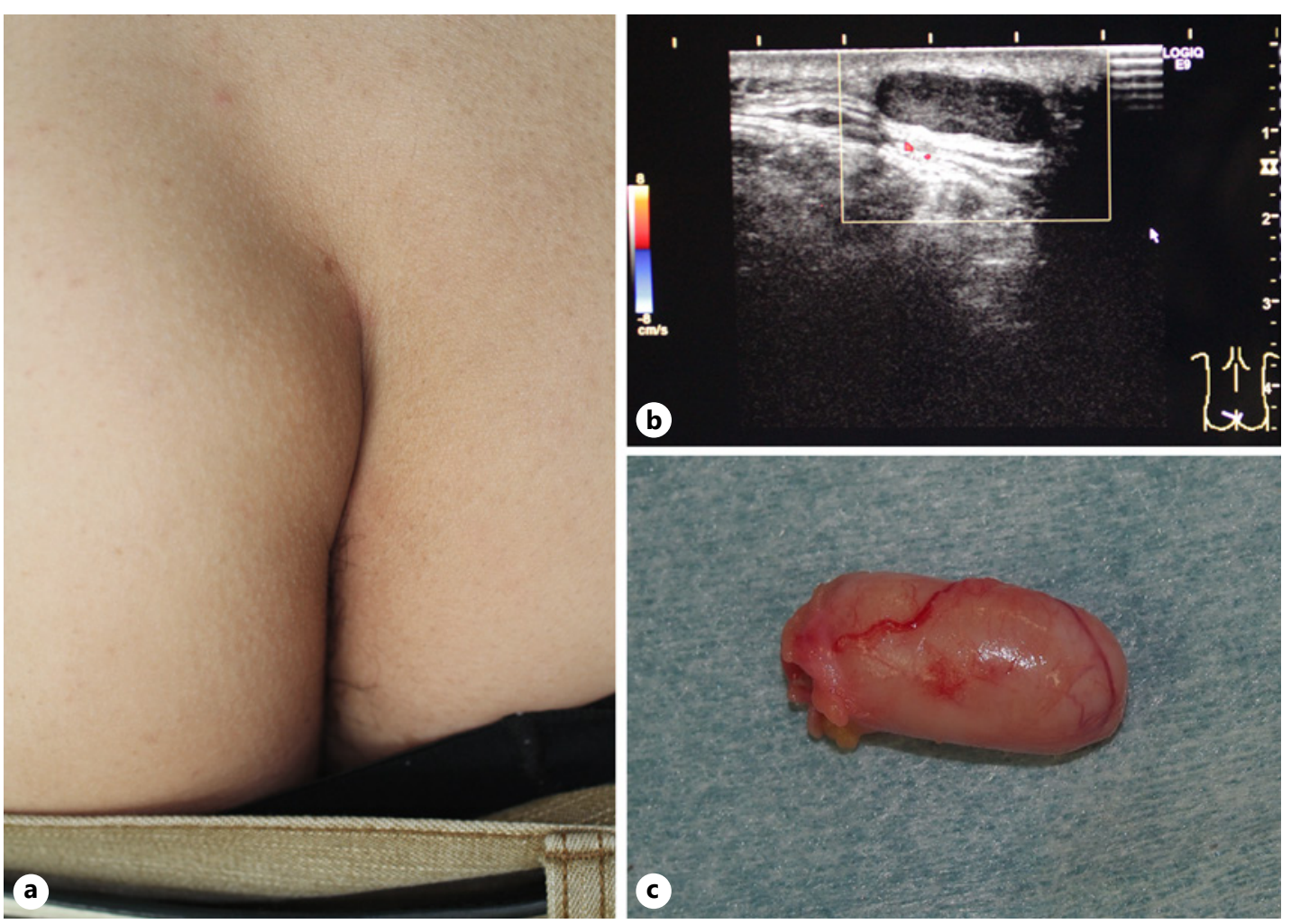

Fig. 1. Clinical appearances of the tumor. a A subcutaneous tumor on the left buttock. $\mathbf{b}$ The image of ultrasonography. c Gross specimen of the excised lesion.

called extraspinal ependymomas, most of which occur in the sacrococcygeal subcutaneous or presacral regions [2]. Extraspinal ependymomas are extremely uncommon tumors and can be misdiagnosed for other benign mass like pilonidal cysts when they occur in the post-sacral area. It is worth noting that they show metastatic potential to distant organs, therefore complete surgical resection is required. The diagnosis is confirmed by histology, and the majority of ependymomas are known to show glial fibrillary acidic protein (GFAP) and S-100 protein immunoreactivity. We herein report a case of GFAP-negative sacrococcygeal subcutaneous extraspinal ependymoma without distant metastases, which presented as an asymptomatic subcutaneous tumor of the left buttock and were clinically misdiagnosed as epidermal cyst.

\section{Case Report}

A Japanese male in his 40s has developed an enlarging, painless, subcutaneous mass on the left buttock over 3 years. The mass had increased in size causing discomfort. There were no other symptoms such as urinary retention or incontinence. Physical examination revealed a mobile soft mass (Fig. 1a). Overlying skin was intact without any color changes. A preoperative ultrasonography showed a $20 \times 16 \times 8 \mathrm{~mm}$ well-circumscribed, hypovascular, and low-echoic lesion with posterior enhancement in the subcutaneous tissue (Fig. 1b). Epidermal cyst and lipoma were considered as differential diagnosis; however, the ultrasonography did not show typical features of both of them. The tumor was completely excised with local anesthesia. The gross specimen showed a well-encapsulated mass with smooth surface (Fig. 1c). Intraoperative findings did not show direct invasion to the sacrococcygeal bones and surrounding musculature. 


\section{Case Reports in Dermatology}
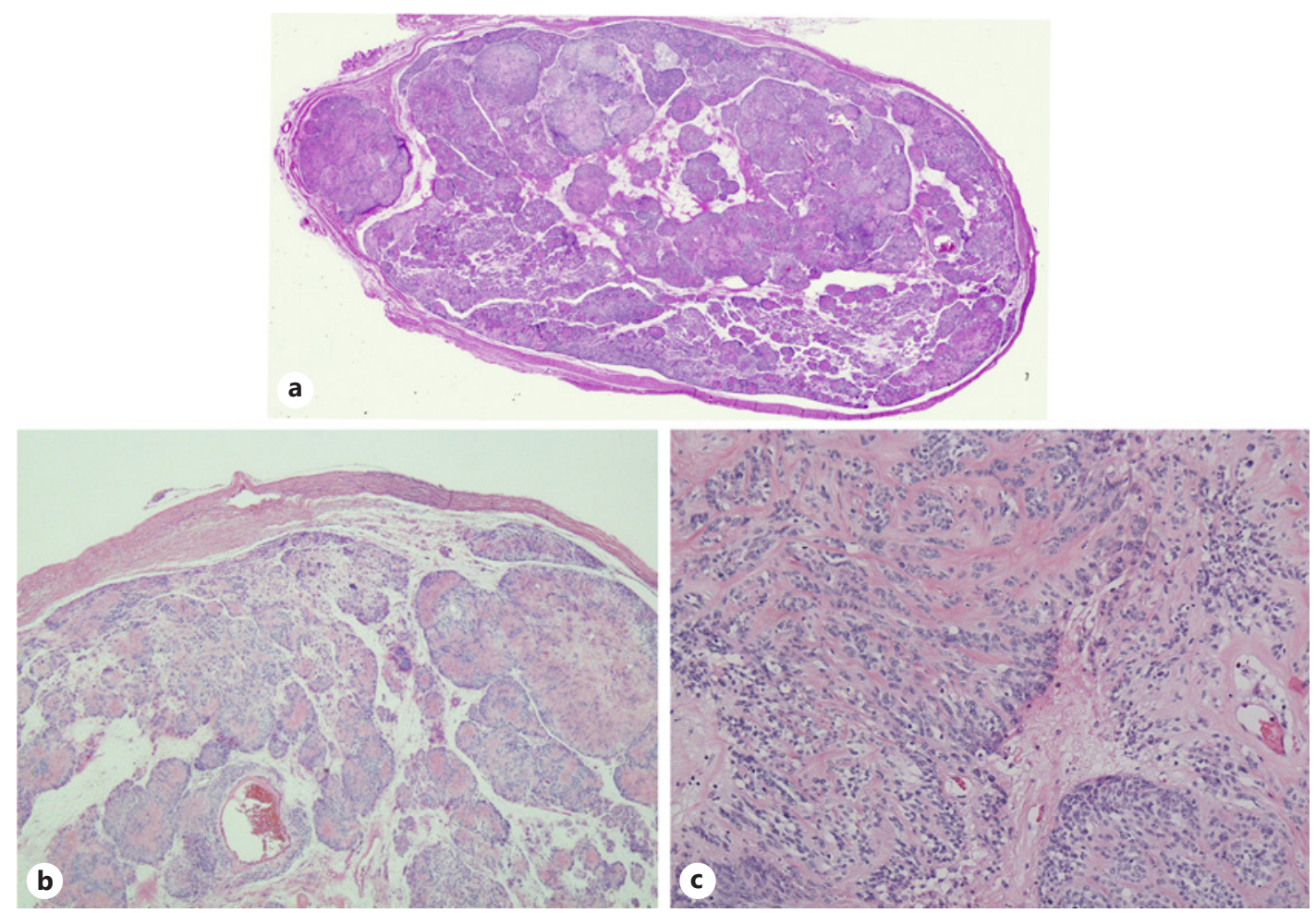

Fig. 2. Histopathology of the lesion (hematoxylin-eosin stain). a The whole tumor covered with fibrous capsule (whole slide image). b Multiple large and small nodules distributed within the tumor (original magnification, $\times 100$ ). c Columnar cells arranged in pseudopapillary architecture (original magnification, $\times 400$ ).

Fig. 3. IHC (S-100 protein). Diffuse expression of S-100 protein in the neoplastic cells. IHC, immunohistochemistry.

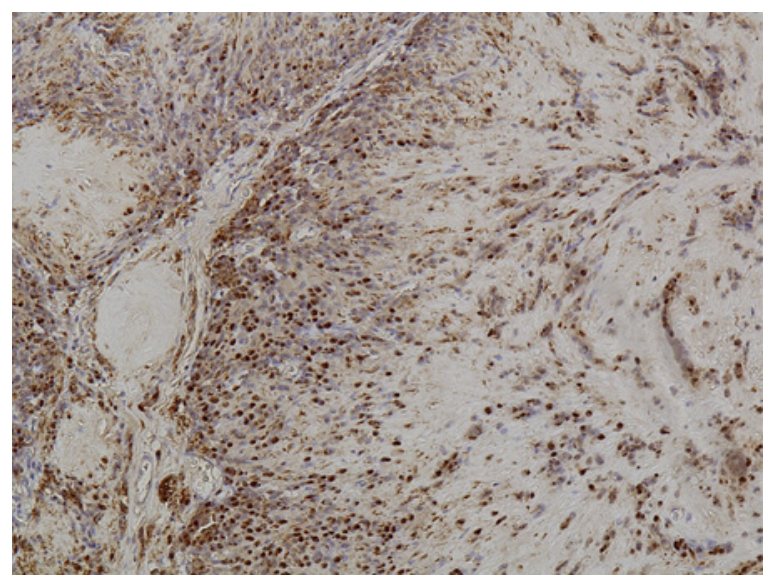

Histologically, whole circumference of this tumor was covered with fibrous capsule (Fig. 2a). Multiple large and small nodules were distributed within the whole tumor (Fig. 2a, b). Each nodule consisted of columnar cells which were arranged in pseudopapillary architecture, located in myxomatous stroma (Fig. 2c). Mitoses and micronecrosis were absent in the specimen.

Immunohistochemically, the neoplastic cells showed diffuse positive reaction with S-100 protein (Fig. 3), which indicates the glial nature of the lesion. On the other hand, the neoplastic cells were negative for GFAP and pancytokeratin markers AE1AE3. Myxomatous stroma showed positive for alcian blue stains. Based on its morphological appearance and S-100 positivity, the tumor was diagnosed as subcutaneous sacrococcygeal myxopapillary ependymoma. 
No direct invasion was observed by the postoperative magnetic resonance imaging (MRI) of the lumbar spine. Moreover, positron emission tomography-computed tomography, contrast-enhanced computed tomography of the body, and MRI of the brain also revealed no residual tumor. His postoperative course was uneventful, and no further treatment was needed. A routine follow-up was planned as there was a possibility of metastases during a long-time follow-up. However, the patient stopped coming to our department a few months after the surgery and could not be followed up.

\section{Discussion}

Extraspinal ependymomas are classified into presacral and post-sacral ependymomas by their relative locations to the sacrum. Mass-effect symptoms or neurological symptoms are frequently found in presacral ependymomas, while post-sacral ependymomas are usually asymptomatic. The posterior subcutaneous location is the most common and typically present as a growing, asymptomatic mass in the intergluteal fold or buttocks such as our case.

The tumor in our case was diagnosed as a myxopapillary ependymoma by histology although immunohistochemistry was negative for GFAP and AE1AE3. Diffuse positivity of S-100 protein, GFAP, and keratin (AE1/3) are pathologic features of ependymomas in immunohistochemistry $[3,4]$. Reported frequencies for them are 60-91, 87-100, and 98\%, respectively [4]. Thus, negative results for GFAP or AE1/3 cannot rule out ependymomas.

Extraspinal ependymomas are at significant risk of metastasis to other organ systems, such as lymph nodes, bones, lungs, and liver; however, they are not likely to disseminate within the CNS [5]. Long-term post-surgery follow-up of up to 10-20 years is needed as metastases can occur in about $20 \%$ of the patients during that period $[1,6]$.

If an MRI scan had been added for further investigation before resection, the possibility of its malignancy could have been suspected. Reportedly, MRI features of myxopapillary ependymomas are as follows: The T1-weighted signal varies from hypointense to hyperintense, while the T2-weighted signal is hyperintense [7]. Tumors also show homogeneous or heterogeneous enhancement within the legions with contrast-enhanced T1 image [7]. In our case, a preoperative MRI study or needle biopsy could be the next step for the correct diagnosis when the preoperative ultrasonography did not show typical features of other common benign tumors.

In conclusion, extraspinal ependymomas, in spite of its rarity, could be considered as one of the differential diagnosis of a sacrococcygeal subcutaneous tumor. When the lesion does not show typical features of other benign tumors on ultrasonography, MRI as the second-line modality should be applied for the correct diagnosis. More careful examinations of similar cases are encouraged in future.

\section{Acknowledgements}

The authors thank Dr. Tomoo Tokime for clinical advice and Ms. Izumi Wakugawa for English proofreading.

\section{Statement of Ethics}

This work was conducted ethically in accordance with the World Medical Association Declaration of Helsinki. Written informed consent was obtained from the patient for publication of this case report and accompanying images.

\section{Karger'}




\section{Conflict of Interest Statement}

The authors have no conflicts of interest to declare.

\section{Funding Sources}

The authors have no funding sources to declare.

\section{Author Contributions}

All named authors fulfill the International Committee of Medical Journal Editors (ICMJE) criteria for authorship for the manuscript, take responsibility for conception, drafting, revising, and have given final approval to the version to be submitted.

\section{References}

1 Ma YT, Ramachandra P, Spooner D. Case report: primary subcutaneous sacrococcygeal ependymoma: a case report and review of the literature. Br J Radiol. 2006 May;79(941):445-7.

2 Lee KJ, Min BW, Seo HJ, Cho CH. Subcutaneous sacrococcygeal myxopapillary ependymoma in asian female:a case report. J Clin Med Res. 2012 Feb;4(1):61-3.

3 Cruz-Sanchez FF, Rossi ML, Hughes JT, Cervos-Navarro J. An immunohistological study of 66 ependymomas. Histopathology. 1988 Oct;13(4):443-54.

4 Vege KD, Giannini C, Scheithauer BW. The immunophenotype of ependymomas. Appl Immunohistochem Mol Morphol. 2000 Mar;8(1):25-31.

5 Chou S, Soucy P, Carpenter B. Extraspinal ependymoma. J Pediatr Surg. 1987 Sep;22(9):802-3.

6 Helwig EB, Stern JB. Subcutaneous sacrococcygeal myxopapillary ependymoma. A clinicopathologic study of 32 cases. Am J Clin Pathol. 1984 Feb;81(2):156-61.

7 Wippold FJ 2nd, Smirniotopoulos JG, Moran CJ, Suojanen JN, Vollmer DG. MR imaging of myxopapillary ependymoma: findings and value to determine extent of tumor and its relation to intraspinal structures. AJR Am J Roentgenol. 1995 Nov;165(5):1263-7. 